\title{
MONG: An extension to Galaxy Clusters
}

\section{Louise Rebecca ${ }^{1}$ Arun Kenath*1,2 C Sivaram $^{3}$}

\begin{abstract}
The presence of dark matter, though well established by indirect evidence is yet to be observed directly. Various dark matter detection experiments running for several years have yielded no positive results so far. In view of these negative results, we had earlier proposed alternate models by postulating a minimum gravitational field strength (minimum curvature) and also a minimum acceleration. These postulates led to the modified Newtonian dynamics and modified Newtonian gravity (MONG). The observed flat rotation curves of galaxies had also been accounted for through these postulates. Here we extend these postulates to galaxy clusters and model the dynamical velocity-distance curves for such large-scale structures. The velocity-distance curve of the Virgo cluster, plotted with this model is found to be in accordance with that observed.
\end{abstract}

Keywords: dark matter; dark energy; modified Newtonian gravity; flat rotation curve; velocity-distance curve

\footnotetext{
*Corresponding author

${ }^{1}$ Department of Physics, Christ Junior College, Bengaluru, 560029, Karnataka, India

${ }^{2}$ Department of Physics and Electronics, CHRIST (Deemed to be University), Bengaluru, 560029, Karnataka, India

${ }^{3}$ Indian Institute of Astrophysics, Bengaluru, 560034, Karnataka, India

E-mail addresses: louise.rheanna@cjc.christcollege.edu (Louise Rebecca); kenath.arun @ cjc.christcollege.edu (Arun Kenath); sivaram@iiap.res.in (C Sivaram)
} 


\section{Introduction}

The nature of dark matter (DM) and dark energy (DE), which constitutes more than 95\% of the energy density of the Universe, is still uncertain. Though there is indirect evidence for the presence of DM in the Universe, its nature and distribution still remain unknown for the most part. Experiments for the detection of DM particles have been running for years with no positive results so far, only lower and lower limits for their fluxes (for different DM particle masses) are set [1]. This perhaps necessitates the need for alternate models to dark matter [2].

Dark matter plays an important role in the observed dynamics of large-scale structures. One of the key evidence of its presence is the rotation curves of galaxies [3]. The manner in which the velocities vary with radius gives the distribution of mass in a galaxy. For a solid disk, the velocity would vary linearly with the radius. If the mass is concentrated at the center of the galaxy, the velocity decreases with the square root of the radius (Keplerian case). The rotation curves of most galaxies have two regions. The inner region is linear, implying that velocities increase linearly with the radius. The outer region, i.e. the one corresponding to distances away from the center is flat suggesting that the velocities remain constant at the outer regions until the visible edge of galaxies. A flat rotation curve thus implies that the mass is still increasing with radius in the outer regions. This led to the conclusion that there is a halo of invisible mass (dark matter) surrounding the galaxy.

\section{Modification of Ne wtonian Gravity (MONG)}

In earlier papers [4-6] we have shown that the flat rotation curves can alternatively be explained by considering Modifications of Newtonian Gravity (MONG) by adding an additional gravitational self-energy density term as well as the dark energy cosmological constant term to the Poisson's equation. Here we extend these considerations (i.e., MONG) to the observed velocity-distance relations for large galaxy clusters such as the Virgo cluster. The gravitational self-energy density also contributes to the gravitational field along with matter density $\rho$. With this modification, we have:

$\nabla^{2} \phi=4 \pi G \rho+K(\nabla \phi)^{2}+\Lambda c^{2}$

Where, $\phi\left(=\frac{G M}{r}\right)$ is the gravitational potential and the constant $K \approx\left(\frac{G^{2}}{c^{2}}\right) \cdot K(\nabla \phi)^{2}$ is the gravitational self-energy density [4]. The matter density is considered to be uniform in the regions closer to the galactic centre up to a distance $r_{o}$ after which it falls off with distance as, $\rho=\rho_{o}\left(\frac{r_{o}}{r}\right)^{2}$ 
For regions within $r_{o}$ - where matter density dominates and is a constant - the equation (1) is the usual Poisson's equation (i.e., $\nabla^{2} \phi=4 \pi G \rho$ ) with the solution $\phi=\frac{G M}{r}$ giving a velocity that varies linearly with distance. At larger distances from the centre of the galaxy, > $r_{0}$, the matter density falls off as equation (2) and the gravitational self-energy dominates. The equation (1) now takes the form (neglecting the constant $\Lambda$ (cosmological constant) term),

$\nabla^{2} \phi=4 \pi G \rho_{o}\left(\frac{r_{o}}{r}\right)^{2}+K(\nabla \phi)^{2}$

The solution of this equation yields:

$\phi=\left(Q+K^{\prime}\right) \ln \frac{r}{r_{o}}$

where $Q=4 \pi G \rho_{o} r_{o}^{2}$ and $K^{\prime} \approx \frac{G M}{r_{o}}$ are constants.

It was also discussed in the recent paper [4] that the regions near the outskirts of a large structure are subjected to minimum gravitational field strength with minimum acceleration given as [7],

$a_{\min }=\frac{G M}{r_{\max ^{2}}}$

Here $r_{\text {max }}$ is the radius of the structure corresponding to the minimum acceleration. This minimum acceleration $\left(a_{\min } \approx 10^{-8} \mathrm{cms}^{-2}\right)$ [7] is of the same order as that of the MOND acceleration [8-10].

Equation (4) gives a force (per unit mass) of the form,

$F=\frac{K^{\prime \prime}}{r}$

where $K^{\prime \prime}=\left(G M a_{\min }\right)^{1 / 2}$ is also a constant.

The balance of the centripetal force and gravitational force gives,

$\frac{V^{2}}{r}=\frac{K^{\prime \prime}}{r}$

Equation (7) gives the relation $v^{2}=K^{\prime \prime}$

Thus implying the independence of velocity $(v)$ on distance $(r)$, as indicated by the flat rotation curve.

For larger distances from the center of the galaxy/cluster (i.e. $\gg r_{o}$ ) the matter density is very small and the dark energy dominates, equation (1) now takes the form

$\nabla^{2} \phi=K(\nabla \phi)^{2}+\Lambda c^{2}$

where, $\Lambda$ is the constant cosmological constant term.

The solution of the above equation yields

$\phi=A \ln s e c B r$ 
where $A \approx\left(G M a_{\text {min }}\right)^{1 / 2}$ and $B \approx \sqrt{\Lambda} c\left(G M a_{\text {min }}\right)^{-1 / 4}$ are constants. This gives a force (per unit mass) of the form

$F=\nabla \phi=B^{\prime} \tan B r$

where $B^{\prime} \approx \sqrt{\Lambda} c\left(G M a_{\text {min }}\right)^{1 / 4}$ is also a constant.

On expanding equation (10) we get,

$F=\nabla \phi=B^{\prime}\left(B r+\frac{1}{3}(B r)^{3}+\cdots\right)$

The outskirts of the large-scale structure are subjected to a minimum acceleration of $10^{-8} \mathrm{cms}^{-2}$. For the Virgo cluster with $M \approx 10^{48} \mathrm{~g}$, the constants take the values of: $B \approx$ $10^{-27} \mathrm{~cm}^{-1}$ and $B^{\prime} \approx 10^{-9} \mathrm{cms}^{-2}$. Hence, at larger distances (outskirts of the cluster) the higher order terms of equation (11) become negligible. The dependence of the constant $B^{\prime}$ on the mass of galaxy cluster is small, even an increase in mass by two orders would yield a value same as above. This gives a force (per unit mass) of the form,

$F=\Lambda c^{2} r$

The distance from the centre of the structure at which the dark energy term starts to dominate can be estimated from equation (12). With the observed cosmological constant term of $\Lambda=10^{-56} \mathrm{~cm}^{-2}$, this distance $(r)$ turns out to be $\approx 10^{26} \mathrm{~cm}$. The dark energy (DE) dominated region can be observed in galaxy clusters rather than individual galaxies as this region is at a much greater distance (at least by two orders) than the typical size of galaxies.

The balance of the centripetal force and (modified) gravitational force now gives, $\frac{V^{2}}{r}=\Lambda c^{2} r$

The above equation implies a linear variation of velocity with distance. Thus, this model predicts an increase in velocity with distance for regions farther away from the centre of the large-scale structures (galaxy clusters).

The constraints on this distance (after which DE dominates) can be set by incorporating the balance of gravitational self-energy and the repulsive dark energy as the boundary condition, i.e.:

$\frac{\left(G M a_{\min }\right)^{\frac{1}{2}}}{r}=\Lambda c^{2} r$

This gives,

$r=\sqrt{\frac{\left(G M a_{\min }\right)^{1 / 2}}{\Lambda c^{2}}}$

Equation (15) gives the distance from the centre of the galaxy/cluster after which the dark energy term dominates. For the Virgo cluster $\left(M \approx 4 \times 10^{48} \mathrm{~g}\right)$, this distance turns out to be 
around $20 M p c$. The velocity variation with distance for Virgo cluster is plotted in Figure 1, the transition (at 20Mpc) to linear increase is in velocity with distance is marked. We see that this is in accordance with observations [11].

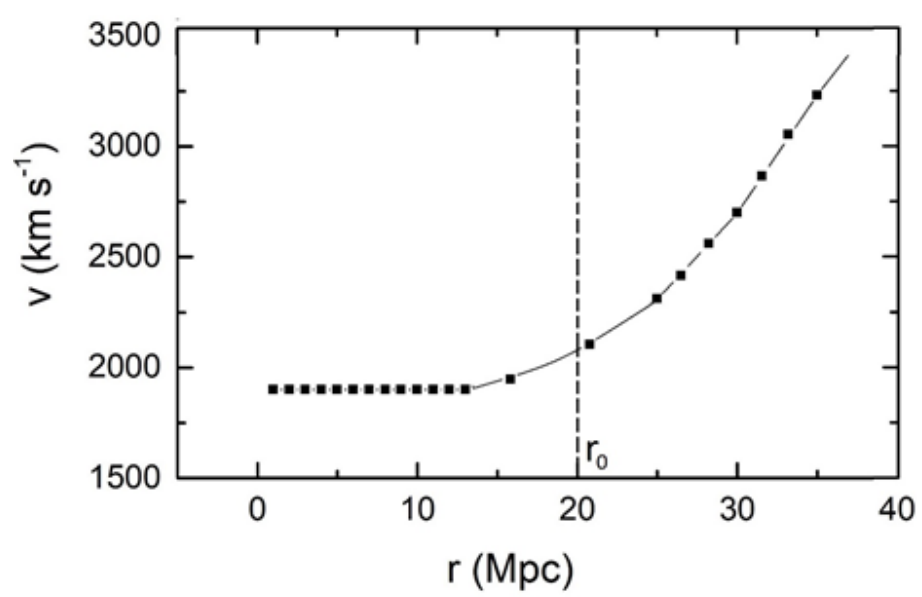

Figure 1 Radial velocity versus distance relation for galaxies in the Virgo cluster

\section{Conclusion}

The addition of the dark energy density and a gravitational self-energy density to the Poisson's equation leads to the Modification of Newtonian Gravity and with the introduction of a minimum acceleration in Newtonian dynamics the flat rotation curves of galaxies clusters is modeled without invoking DM. The region close to the center of the galaxy cluster, where the matter density dominates and is uniform i.e., $<r_{0}$, gives a linear increase in velocity with distance.

For distances $>r_{0}$, the matter density falls off with distance and the gravitational selfenergy term dominates giving a velocity that is independent of distance implying a flat region in the rotation curve, thereby accounting for dark matter (DM). The regions at the outskirts of the galaxy cluster (where the dark energy dominates) are subjected to a minimum gravitational field strength and a minimum acceleration $\left(a_{0} \approx 10^{-8} \mathrm{cms}^{-2}\right)$ which was introduced in an earlier work to avoid an ad hoc fundamental acceleration accounting for dark matter. As dark energy begins to dominate, the velocity is found to increase linearly with distance. In the case of the Virgo cluster, the dark energy is found to dominate from a distance of 20Mpc (Fig. 1) where the curve shifts from being flat to linear which is found to be consistent with observations. The DM effects can be, in principle and at least partially, be explained through the framework of extended gravity which has been considered earlier [12, 13]. 


\section{References:}

[1] E. Aprile, et al., Dark matter search results from a one ton-year exposure of XENON1T, Phys. Rev. Lett. 121 (2018) 111302.

[2] K. Arun, S.B. Gudennavar, C. Sivaram, Dark matter, dark energy and alternate models: A review, Adv. Space Res. 60 (2017) 166.

[3] F. Zwicky, On the masses of nebulae and of clusters of nebulae, Astrophys. J. 86 (1937) 217.

[4] C. Sivaram, K. Arun, L. Rebecca, MOND, MONG, MORG as alternatives to dark matter and dark energy, and consequences for cosmic structures, J. Astron. Astrophys. 41 (2020) 4. [5] L. Rebecca, K. Arun, C. Sivaram, Dark matter density distributions and dark energy constraints on structure formation including MOND, Indian J. Phys. 94 (2020) 1491.

[6] L. Rebecca, K. Arun, C. Sivaram, Dark energy constraints on masses and sizes of large scale cosmic structures, Astrophys. Space Sci. 363 (2018) 149.

[7] V. de Sabbata, C. Sivaram, On limiting field strengths in gravitation, Found. Phys. Lett. 6 (1993) 561.

[8] M. Milgrom, A modification of the Newtonian dynamics as a possible alternative to the hidden mass hypothesis, Astrophys. J. 270 (1983) 365.

[9] M. Milgrom, MOND vs. dark matter in light of historical parallels, 2019, ArXiv e-prints, arXiv:1910.04368 [astro-ph.GA].

[10] C. Sivaram, Some aspects of MOND and its consequences for cosmology, Astrophys. Space Sci. 215 (1994) 185.

[11] I.D. Karachentsev, O.G. Nasonova, The observed infall of galaxies towards the Virgo cluster, Mon. Not. R. Astron. Soc. 405 (2010) 1075.

[12] C. Corda, Interferometric detection of gravitational waves: the definitive test for General Relativity, Int. J. Mod. Phys. D 18 (2009) 2275.

[13] C. Corda, The future of gravitational theories in the era of the gravitational wave astronomy, Int. J. Mod. Phys. D 27 (2018) 1850060. 